\title{
Combination of L-Carnitine and Angiotensin-II type 1 Receptor Blocker has Beneficial Effects on Hepatic Fibrosis in a Non-Alcoholic Steatohepatitis Rat Model
}

\author{
Takuya Kubo, Hideto Kawaratani*, Yasuhiko Sawada, Yukihisa Fujinaga, Takahiro Ozutsumi, Daisuke \\ Kaya, Yuki Tsuji, Keisuke Nakanishi, Masanori Furukawa, Kou Kitagawa, Soichiro Saikawa, Shinya Sato, \\ Hiroaki Takaya, Kosuke Kaji, Naotaka Shimozato, Kei Moriya, Tadashi Namisaki, Takemi Akahane, \\ Akira Mitoro and Hitoshi Yoshiji \\ Department of Gastroenterology, Nara Medical University, Japan \\ *Corresponding author: Hideto Kawaratani, Department of Gastroenterology, Nara Medical University, Japan
}

\begin{abstract}
Inflammation and oxidative stress contribute to the progression of nonalcoholic steatohepatitis (NASH). Hepatic fibrosis and activated hepatic stellate cells (Ac-HSCs) are attenuated by Angiotensin-II type 1 Receptor Blocker (ARB), and L-carnitine is effective for NASH by ameliorating oxidative stress, but neither agent is effective in a clinical setting. We evaluated the effect of the combination of L-carnitine and ARB on liver fibrosis using a rat NASH model. A Choline-Deficient/L-Amino Acid-defined (CDAA) diet was fed to F344 rats for 8 weeks. The rats were then divided into a control group, group receiving L-carnitine or ARB alone, and group receiving L-carnitine plus ARB. Therapeutic efficacy was assessed by evaluating liver fibrosis, liver fatty acid metabolism, and oxidative stress. ARB inhibited liver-specific tumor necrotic factor- $\alpha$ and LPS-binding protein, which are involved in hepatic inflammation. L-Carnitine reduced hepatic oxidative stress by rescuing hepatic sterol-regulatory elementbinding protein 1 and thiobarbituric acid reactive substances induced by the CDAA diet. Combination of L-carnitine and ARB improved liver fibrosis, with concomitant HSC suppression. Therefore, we suggest that L-carnitine and ARB are effective in suppressing liver fibrosis. Currently both drugs are in clinical use, and a combination of the two could be an effective therapy for NASH fibrosis.
\end{abstract}

Keywords: Angiotensin-2 type 1 Receptor Blocker; L-carnitine; Nonalcoholic Steatohepatitis; Hepatic Fibrosis; Oxidative Stress

\section{Introduction}

Non-Alcoholic Fatty Liver Disease (NAFLD) is the most common liver disorder in developed countries. It is divided into simple fatty liver and non-alcoholic steatohepatitis (NASH), which involves liver inflammation. NASH is a progressive liver disease leading to hepatic fibrosis, cirrhosis, and cancer, and it does not have an established treatment. The pathogenesis of NASH is like that of alcoholic steatohepatitis, with a two-step process beginning with excessive fat accumulation in the liver, followed by aggravating factors such as inflammatory cytokines, oxidative stress, and endotoxins [1]. However, it has also been suggested that inflammatory cytokines, oxidative stress, endotoxins, and other factors are contributing factors [2]. NAFLD progression is dependent on both genetic and environmental factors [2,3], including non-synonymous SingleNucleotide Polymorphisms (SNPs) in PNPLA3 and TM6SF2 [4,5], as well as oxidative stress and inflammation.

L-Carnitine (4-N-trimethylammonium-3-hydroxybutyric acid), which is involved in $\beta$-oxidation of fatty acids, is a nutrient conditionally synthesized from methionine and lysine in the brain, liver, and kidneys, especially, the liver is the main tissue for carnitine synthesis [6], and is mainly obtained from meat and dairy products [7]. It is an accepted treatment for mitochondrial myopathy and encephalomyopathy as well as other states of primary and 
secondary carnitine deficiencies [8] and has recently been applied to treat hepatic encephalopathy.

On the contrary, the renin-angiotensin-aldosterone system plays an important role in chronic liver disease $[9,10]$. We previously reported that blocking angiotensin-II (AT-II) signaling via the AT-II type 1 receptor (AT1R) suppresses liver fibrosis in rats $[11,12]$. Furthermore, the inhibitory effect of Angiotensin-2 Type 1 Receptor Blocker (ARB) on hepatic fibrosis is consistent with the suppression of activated hepatic stellate cells [13]. In addition, ARB improves liver fibrosis via AT-II-mediated LPS-toll-like receptor 4 (TLR4) signaling and suppresses TLR4 signaling in Ac-HSCs [14]. We hypothesize that L-carnitine might improve the progression of NASH by inhibiting oxidative stress, and its use in combination with ARB may inhibit the development of NASH fibrosis via various mechanisms. In this study, we examined the effect of the combination of L-carnitine and ARB on NASH fibrosis using a rat model fed a Choline-Deficient/L-Amino Acid-defined (CDAA) diet.

\section{Materials and Methods}

\section{Animals and Regents}

Male 6-week-old Fisher 344 (F344) rats were purchased from Japan SLC (Hamamatsu, Shizuoka, Japan). L-Carnitine was purchased from Otsuka Pharmaceutical Co. Ltd. (Tokyo, Japan). Losartan was purchased from Merck Co., Ltd. (Tokyo, Japan). Conventional chemical reagents were purchased from Funakoshi (Tokyo, Japan). CDAA and choline-supplemented/L-amino acid-defined (CSAA) diets were purchased from CLEA Japan Inc. (Tokyo, Japan).

\section{Experimental Design}

After one week of acclimatization, 30 rats weighing 160-175 g were randomly divided into five groups. A control group that was fed the CSAA diet for normal non-NASH baseline reference. Four other groups were provided the CDAA diet to establish diet-induced hepatic steatosis and fibrosis, which simulates human NASH. One group received no further treatment, while the second group received additional L-carnitine at $200 \mathrm{mg} / \mathrm{kg} /$ day and the third group received the ARB losartan at $30 \mathrm{mg} / \mathrm{kg} /$ day. Finally, a combination treatment group received both L-carnitine and losartan. These treatment conditions were maintained for 8 weeks, during which the rats had free access to tap water. At the end of the experimental period, the rats were anesthetized with pentobarbital and sacrificed, and the liver samples were collected. All animal procedures were performed in accordance with the Declaration of Helsinki and in compliance with the standard recommendations for the proper care and use of laboratory animals. The protocol was approved by the Animal Care and Use Committee of Nara Medical University.

\section{Histological and Immunohistochemical Analyses}

Five-micrometer-thick sections of formalin-fixed and paraffinembedded liver specimens were stained using hematoxylin and eosin and Azan stains to evaluate fibrosis. Besides, immunohistochemical staining of alpha smooth muscle actin ( $\alpha$-SMA; DAKO, Kyoto, Japan), which correlates with Ac-HSCs, was performed as previously described $[15,16]$. The stained sections were analyzed using Adobe Photoshop software ver. 6 (Adobe, Tokyo, Japan).

\section{Quantitative RT-PCR Analysis}

mRNA was extracted from frozen liver tissues using the RNeasy Mini Kit (QIAGEN, Tokyo, Japan). The total RNA (1 $\mu \mathrm{g})$ extracted from each sample was reverse transcribed into cDNA using a high capacity RNA-to-cDNA kit (Applied Biosystems Inc., Foster City, CA, USA). As TNF- $\alpha$ correlates with hepatic inflammation, it leads to liver fibrosis. As direct detection of LPS is difficult, we evaluated hepatic LPS-binding protein (LBP) because this directly correlates with LPS [17]. LPS stimulates TLR4 and activates Kupffer cells, which leads to hepatic inflammation. Hepatic oxidative stress was evaluated using sterol-regulatory element-binding protein 1 (SREBP-1) mRNA. The expression of mRNA encoding liver tissuederived TNF- $\alpha$, LBP, and SREBP1 was analyzed using the PowerUp SYBR Green Master Mix and Step One Sequence Detection System (Applied Biosystems Inc., Foster City, CA, USA) by qRT-PCR. The PCR conditions were as follows: $95^{\circ} \mathrm{C}$ for $20 \mathrm{~s}$, and 40 cycles at $95^{\circ} \mathrm{C}$ for $3 \mathrm{~s}$ and $60^{\circ} \mathrm{C}$ for $30 \mathrm{~s}$. $\beta$-Actin was used as the endogenous control. The sequence of primers used is shown in Table 1.

Table1: Primer sequences for qRT-PCR.

\begin{tabular}{|c|c|c|}
\hline \multirow{2}{*}{ TNF- $\alpha$} & $\begin{array}{c}\text { Forward } \\
\text { Reverse }\end{array}$ & $\begin{array}{r}\text { 5'-ACT CCC AGA AAA GCA AGC AA-3' } \\
\text { 5'-CGA GCA GGA ATG AGA AGA GG-3' }\end{array}$ \\
\hline \multirow{2}{*}{ LBP } & Forward & 5'-ACT CCC AGA AAA GCA AGC AA-3' \\
& Reverse & 5'-CGA GCA GGA ATG AGA AGA GG-3' \\
\hline \multirow{2}{*}{ SREBP1 } & Forward & 5'-GTG GTC TTC CAG AGG CTG AG-3' \\
& Reverse & 5'-GGG TGA GAG CCT TGA GAC AG-3' \\
\hline \multirow{2}{*}{$\beta$-actin } & Forward & 5'-GGA GAT TAC TGC CCT GGC TCC TA-3' \\
& Reverse & 5'-GAC TCA TCG TAC TCC TGC TTG CTG-3' \\
\hline
\end{tabular}

\section{Measurement of Thiobarbituric Acid Reactive Substances (TBARS)}

We evaluated hepatic oxidative stress using hepatic TBARS concentration. Liver homogenates were prepared with PBS using a tissue homogenizer (Power Masher IIß; Nippi, Tokyo, Japan), and then protein was extracted. TBARS concentration was measured using a commercially available kit, with malondialdehyde as the standard (Cayman Chemical, MI, USA).

\section{Statistical Analyses}

All results are expressed as mean \pm SD. Statistical analyses were performed using EZR ver. 1.40 (Saitama Medical Center, Jichi Medical University). All tests were two-tailed and the results with $\mathrm{p}$ values $<0.05$ were considered statistically significant. 


\section{Results}

\section{Inhibitory Effect of L-Carnitine and ARB on Hepatic Fibrosis}

The groups fed the CDAA diet showed activated liver fibrosis compared with that of the group fed the CSAA diet. In the L-carnitine group, liver fibrosis was slightly improved compared with that in the CDAA-only group ( $p=0.07)$, and significantly improved in the ARB group ( $p<0.05)$. The combination of L-carnitine and ARB resulted in a greater improvement than with either drug alone (Figure 1A \& 1B). Immunohistochemistry of $\alpha$-SMA indicated a significant decrease in $\alpha$-SMA-immunopositive Ac-HSCs in groups treated with ARB (Figure 2A). The semiquantitative analysis of $\alpha$-SMA immunohistochemistry showed reduced $\alpha$-SMA positive staining along with the inhibition of hepatic fibrosis (Figure 2B). A significant inhibition of TNF- $\alpha$ mRNA expression was also observed in the ARB group compared with that in the CDAA group (Figure 3A) $(\mathrm{p}<0.05)$. However, L-carnitine did not inhibit TNF- $\alpha$ mRNA expression compared with that in the CDAA group. The combination of L-carnitine and ARB resulted in an inhibitory effect equal to that of $\mathrm{ARB}$ alone.

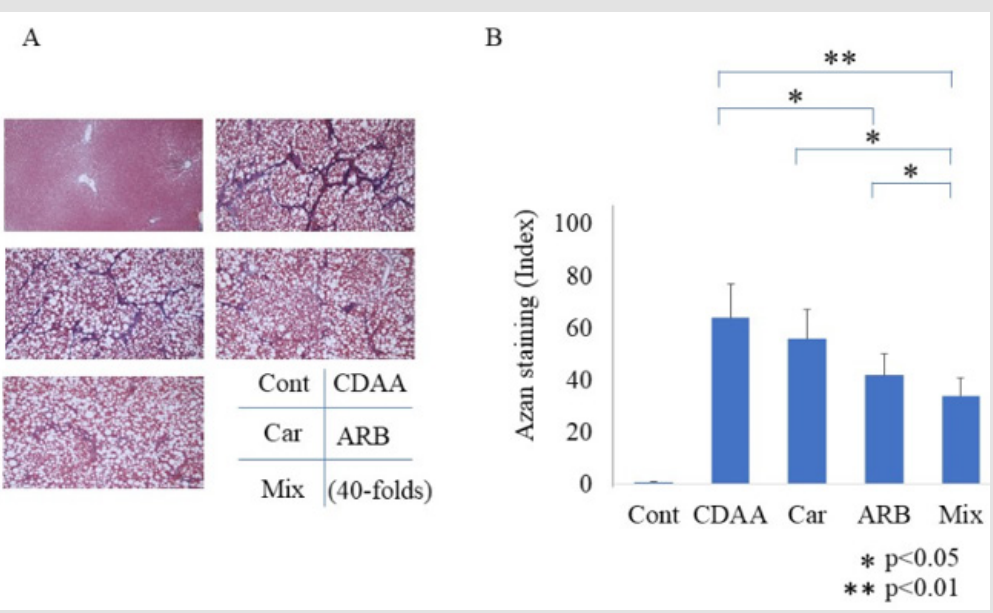

Figure 1: Azan staining of liver sections from non-alcoholic steatohepatitis model mice. Presented images are representative of multiple images taken. No fibrosis was observed in the control group. Liver fibrosis was observed in the group that was fed a Choline-Deficient L-Amino Acid (CDAA) diet. Monotherapy with L-Carnitine (Car) induced a small inhibitory effect compared to that in the CDAA group $(\mathrm{P}=0.07)$. Monotherapy with an Angiotensin II Type I Receptor Blocker $(\mathrm{ARB})$ resulted in a significant inhibitory effect $(\mathrm{p}<0.05)$. Combination therapy with L-carnitine and ARB (Mix) exerted a greater inhibitory effect than that with either monotherapy. ${ }^{*} \mathrm{P}<0.05, * * \mathrm{P}<0.01$.

A. Microphotographs of Azan-stained liver sections (40-fold).

B. A semi-quantitative analysis of Azan-stained sections.

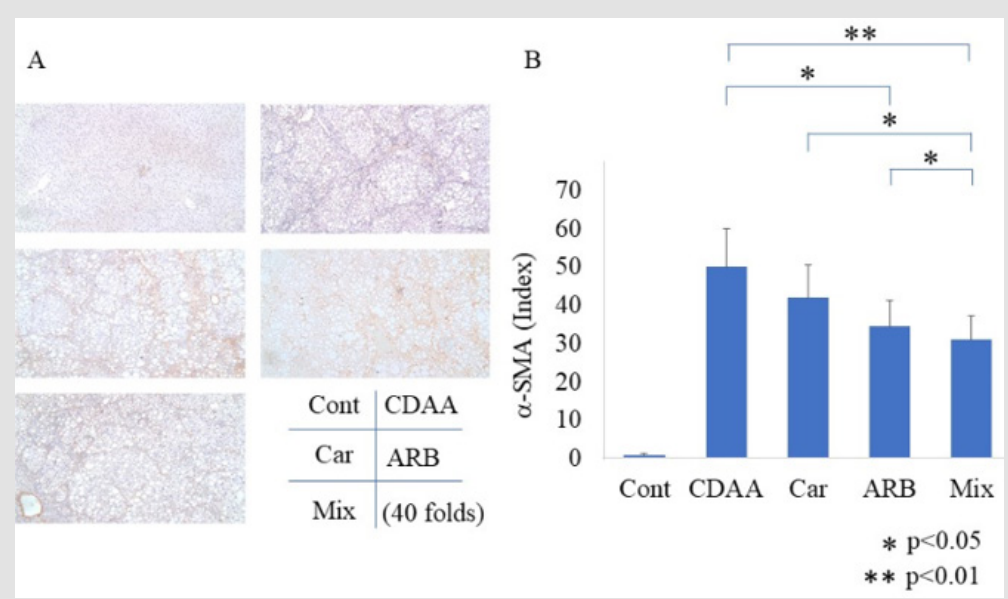

Figure 2: Alpha-smooth muscle actin expression in non-alcoholic steatohepatitis model mice. Presented images are a representative image of multiple images taken. No a-SMA-positive cells were observed in the liver sections from the control group. The choline-deficient L-amino acid (CDAA) group showed a-SMA-positive cells. Treatment with L-Carnitine (Car) induced a small inhibitory effect $(\mathrm{P}=0.08)$. Treatment with an Angiotensin II Type I Receptor Blocker $(\mathrm{ARB})$ resulted in a greater inhibitory effect on hepatic a-SMA expression in comparison to that in the CDAA group. Combination treatment with L-Carnitine and ARB (Mix) exerted the strongest inhibitory effect of all treatment regimes. ${ }^{*} \mathrm{P}<0.05,{ }^{* *} \mathrm{P}<0.01$.

A. Immunohistochemistry of liver sections stained for hepatic a-smooth muscle actin (a-SMA; 40-fold).

B. A semi-quantitative analysis of a-SMA expression. 

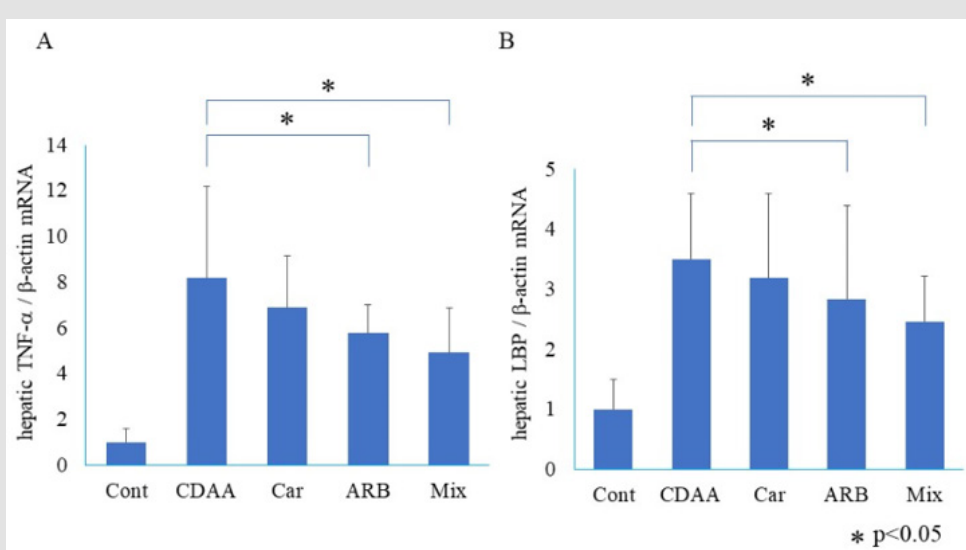

Figure 3: Markers of inflammation in nonalcoholic steatohepatitis mouse model. Hepatic TNF- $\alpha$ and LBP mRNA levels were markedly increased in the Choline-Deficient L-Amino Acid (CDAA) treatment group in comparison to those in the control group. The administration of L-Carnitine (Car) did not affect the mRNA expression of TNF- $\alpha$ and LBP, relative to that in the CDAA group. The administration of an Angiotensin II Type I Receptor Blocker (ARB) significantly suppressed hepatic TNF- $\alpha$ and LBP mRNA expression in comparison to that in the CDAA group. Treatment with a combination of L-carnitine and ARB (Mix) resulted in a response that was equivalent to that of L-carnitine monotherapy in terms of TNF- $\alpha$ and LBP mRNA. The combination of L-carnitine and ARB (Mix) exerted a greater inhibitory effect than either monotherapy on $\alpha$-SMA mRNA. Values represent the mean $\pm \mathrm{SD} .{ }^{*} \mathrm{P}<0.05, * * \mathrm{P}<0.01$.

A. Semi-quantification of hepatic TNF- $\alpha$ mRNA expression by RT-PCR.

B. Semi-quantification of hepatic LBP mRNA expression by RT-PCR

\section{Effect of L-Carnitine and ARB on LPS Signaling}

Hepatic LBP mRNA expression increased in the CDAA group compared with that in the CSAA group (Figure 3B). In the ARB and combination treatment groups, hepatic LBP mRNA expression was significantly lower than that in the CDAA group $(\mathrm{p}<0.05)$. Hepatic LBP mRNA expression in the L-carnitine group was slightly lower, but not significantly different.

\section{Inhibitory Effect of L-Carnitine and ARB on Oxidative Stress}

Liver SREBP1 mRNA expression increased significantly over the control after 8 weeks of CDAA feeding ( $p<0.01)$. In the L-carnitine group, SREBP1 mRNA expression was lower than that in the CDAA group ( $\mathrm{p}<0.05$; Figure $4 \mathrm{~A}$ ), but no significant effect was observed in the ARB group. Under treatment with both L-carnitine and ARB, SREBP1 mRNA expression was lower than that in the CDAA group, but not significantly different from that with L-carnitine alone. Similarly, the increase in hepatic TBARS level in mice fed the CDAA diet, was decreased by L-carnitine treatment $(p<0.05$; Figure $4 B$ ) but did not change under ARB treatment. The effect of combination of L-carnitine and ARB was similar to that of L-carnitine treatment. These data suggest that the inhibitory effect of the combination treatment on hepatic oxidative stress is associated with L-carnitine rather than ARB.

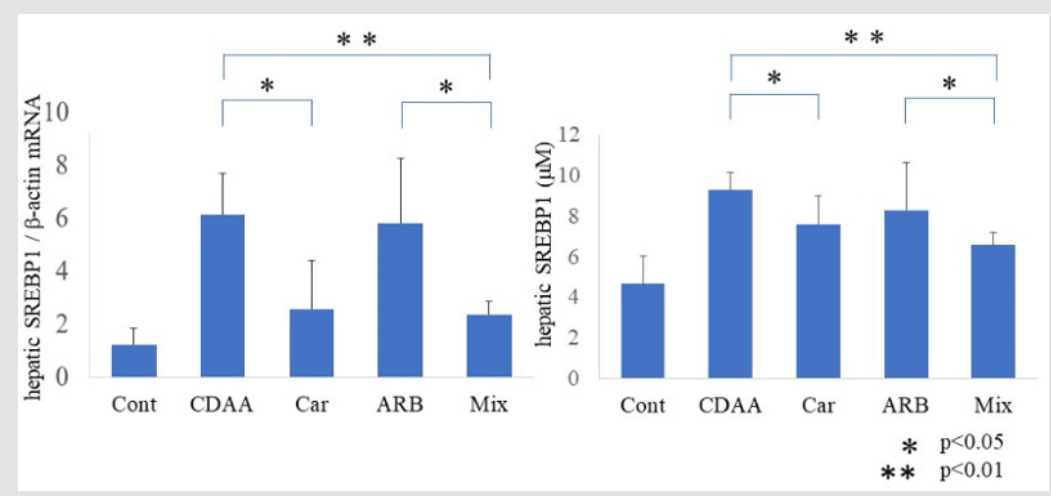

Figure 4: Oxidative stress in a mouse model of non-alcoholic hepatic steatohepatitis. Hepatic SREBP1 mRNA expression and TBARS concentrations were markedly increased in the Choline-Deficient L-Amino Acid (CDAA) group in comparison to that in the control group. The administration of L-Carnitine (Car) significantly reduced both hepatic SREBP1 mRNA expression and TBARS concentrations in comparison to that in the CDAA group. The administration of an Angiotensin II Type I Receptor Blocker (ARB) did not change either measurements relative to those in the CDAA group. The effects of the L-carnitine and ARB (Mix) combination therapy were comparable to those of treatment with L-carnitine alone. Values represent the mean \pm SD. ${ }^{*} \mathrm{P}$ $<0.05, * * \mathrm{P}<0.01$.
A. Semi-quantification of hepatic SREBP1 mRNA by RT-PCR.
B. Quantification of hepatic TBARS concentrations. 


\section{Discussion}

Treatment with L-carnitine and ARB ameliorated liver fibrosis and suppressed Ac-HSCs and oxidative stress in the rat NASH model. Previously, we reported that a CDAA diet caused liver fibrosis by increasing TNF- $\alpha$ [18]. In the present study, the combination treatment with L-carnitine and ARB resulted in a synergistic antifibrotic effect greater than that resulting from either drug alone. L-Carnitine improved hepatic oxidative stress, whereas ARB suppressed the activation of HSCs. NASH has various causative factors, including inflammation and oxidative stress. Activation of HSCs leads to liver inflammation and ultimately fibrosis, which is governed by a complex network of autocrine/paracrine fibrogenic signals promoted by the activation of HSCs and characterized by the expression of $\alpha$-SMA. The interaction between AT-II and AcHSC plays an important role in liver fibrogenesis. We previously reported that ARB directly inhibits Ac-HSC activation and that AT-II is important for the upregulation of TLR4 expression via stimulation of AT1R in Ac-HSCs [14]. Our results showed that ARB treatment of rats with NASH reduces $\alpha$-SMA-positivity and TNF- $\alpha$, which leads to an improvement in hepatic inflammation and fibrogenesis.

L-Carnitine is a vitamin-like dietary compound, synthesized from the essential amino acids lysine and methionine [19]. The liver is the main tissue for carnitine synthesis [6]. L-Carnitine is important for the transport of long chain fatty acids into the mitochondrial matrix via specialized acyltransferases [19]. Thus, L-carnitine inhibits oxidative stress $[20,21]$ and stimulates the $\beta$-oxidation of fatty acids. Under L-carnitine deficiency, mitochondrial fatty acid oxidation is impaired, and lipids accumulate in hepatocyte cytoplasm, leading to the impairment of hepatic functions [22]. Our results showed that L-carnitine treatment reduces hepatic SREBP and TBARS in a rat model of NASH, leading to an improvement in hepatic oxidative stress. In contrast, hepatic TNF- $\alpha$, LBP, and $\alpha$-SMA were unchanged by L-carnitine treatment. Several studies have indicated the effectiveness of L-carnitine for liver oxidative stress and inflammation; however, its effectiveness for liver fibrosis is not known [23,24].

In this study, we examined the inhibitory effect of the combination of L-carnitine and ARB on liver fibrosis. As previously reported, ARB improved only liver fibrosis. Furthermore, L-carnitine slightly improved liver fibrosis without a significant difference. However, the combination of L-carnitine and ARB resulted in a significant improvement in liver fibrosis compared with that of ARB alone. Overall, the combination of L-carnitine and ARB showed a synergistic effect on liver fibrosis. This study was notably limited using only one model animal. It is also difficult to detect the signal transduction of L-carnitine for liver fibrogenesis using in vitro study, as it does not directly affect hepatic parenchymal cells. In conclusion, we found that simultaneous administration of L-carnitine and ARB exerted a potent and synergistic inhibitory effect on hepatic fibrosis compared with either agent alone by suppressing oxidative stress and Ac-HSC proliferation, respectively. This combination therapy could be useful for inhibiting NASH progression in clinical applications.

\section{Acknowledgment}

We would like to thank Editage (www.editage.com) for English language editing.

\section{Conflicts of Interest}

The authors declare no conflict of interest.

\section{References}

1. Day CP, James OF (1998) Steatohepatitis: a tale of two "hits"? Gastroenterology 114(4): 842-845.

2. Tilg H, Moschen AR (2010) Evolution of inflammation in nonalcoholic fatty liver disease: the multiple parallel hits hypothesis. Hepatology 52(5): 1836-1846.

3. Musso G, Gambino R, Cassader M (2010) Gut microbiota as a regulator of energy homeostasis and ectopic fat deposition: mechanisms and implications for metabolic disorders. Curr Opin Lipidol 21(1): 76-83.

4. Rotman Y, Koh C, Zmuda JM, Kleiner DE (2010) The association of genetic variability in patatin-like phospholipase domain-containing protein 3 (PNPLA3) with histological severity of nonalcoholic fatty liver disease. Hepatology 52(3): 894-903

5. Dongiovanni P, Petta S, Maglio C (2015) Transmembrane 6 superfamily member 2 gene variant disentangles nonalcoholic steatohepatitis from cardiovascular disease. Hepatology 61(2): 506-514.

6. Ali SA, Faddah L, Abdel-Baky A, Bayoumi A (2010) Protective effect of L-carnitine and coenzyme Q10 on $\mathrm{CCl}_{4}$-induced liver injury in rats. Sci Pharm 78(4): 881-896.

7. Rebouche CJ, Seim H (1998) Carnitine metabolism and its regulation in microorganisms and mammals. Annu Rev Nutr 18: 39-61.

8. Longo N, Amat di San Filippo C, Pasquali M (2006) Disorders of carnitine transport and the carnitine cycle. Am J Med Genet C Semin Med Genet $142 \mathrm{C}(2): 77-85$

9. Yoshiji H, Kuriyama S, Noguchi R, Fukui H (2004) Angiotensin-I converting enzyme inhibitors as potential anti-angiogenic agents for cancer therapy. Curr Cancer Drug Targets 4(7): 555-567.

10. Yoshiji H, Yoshii J, Ikenaka Y, Noguchi R, Tsujinoue H, et al. (2002) Inhibition of renin-angiotensin system attenuates liver enzyme-altered preneoplastic lesions and fibrosis development in rats. J Hepatol 37(1): $22-30$

11. Yoshiji H, Kuriyama S, Yoshii J, Ikenaka Y, Noguchi R, et al. (2001) Angiotensin-II type 1 receptor interaction is a major regulator for liver fibrosis development in rats. Hepatology 34: 745-750.

12. Yoshiji H, Noguchi R, Ikenaka Y, Namisaki T, Kitade M, et al. (2009) Losartan, an angiotensin-II type 1 receptor blocker, attenuates the liver fibrosis development of non-alcoholic steatohepatitis in the rat. BMC Res Notes 2: 70 .

13. Yoshiji H, Noguchi R, Ikenaka Y, Kaji K, Aihara Y, et al. (2011) Cocktail therapy with a combination of interferon, ribavirin and angiotensin-II type 1 receptor blocker attenuates murine liver fibrosis development. Int J Mol Med 28(1): 81-88.

14. Shirai Y, Yoshiji H, Noguchi R, Kaji K, Aihara Y et al. (2013) Cross talk between toll-like receptor-4 signaling and angiotensin-II in liver fibrosis development in the rat model of non-alcoholic steatohepatitis. J Gastroenterol Hepatol 28(4): 723-730.

15. Kawaratani H, Tsujimoto T, Kitazawa T (2011) Therapeutic effects of cytokine modulator Y-40138 in the rat alcoholic liver disease model. J Gastroenterol Hepatol 26(4): 775-783. 
16. Noguchi R, Yoshiji H, Ikenaka Y, Kaji K, Aihara Y et al. (2013) Dual blockade of angiotensin-II and aldosterone suppresses the progression of a non-diabetic rat model of steatohepatitis. Hepatol Res 43(7): 765774.

17. Du Plessis J, Vanheel H, Janssen CE, Roos L, Slavik T, et al. (2013) Activated intestinal macrophages in patients with cirrhosis release NO and IL-6 that may disrupt intestinal barrier function. J Hepatol 58(6): 1125-1132.

18. Kawaratani H, Tsujimoto T, Kitazawa T (2008) Innate immune reactivity of the liver in rats fed a choline-deficient L-amino-acid-defined diet. World J Gastroenterol 14(43): 6655-6661.

19. Ozsoy SY, Ozsoy B, Ozyildiz Z, Aytekin I (2011) Protective effect of L-carnitine on experimental lead toxicity in rats: a clinical, histopathological and immunohistochemical study. Biotech Histochem 86(6): 436-443.

20. Annadurai T, Vigneshwari S, Thirukumaran R, Thomas PA, Geraldine $P$ (2011) Acetyl-L-carnitine prevents carbon tetrachloride-induced

\section{ISSN: 2574-1241}

DOI: $10.26717 /$ BJSTR.2019.23.003969

Hideto Kawaratani. Biomed J Sci \& Tech Res

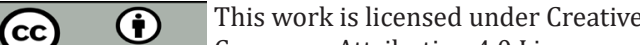

Submission Link: https://biomedres.us/submit-manuscript.php oxidative stress in various tissues of Wistar rats. J Physiol Biochem 67(4): 519-530.

21. Shaker ME, Houssen ME, Abo-Hashem EM, Ibrahim TM (2009) Comparison of vitamin E, L-carnitine and melatonin in ameliorating carbon tetrachloride and diabetes induced hepatic oxidative stress. J Physiol Biochem 65(3): 225-33.

22.Xia Y, Li Q Zhong W, Dong J (2011) L-carnitine ameliorated fatty liver in high-calorie diet/STZ-induced type 2 diabetic mice by improving mitochondrial function. Diabetol Metab Syndr 15: 3-31.

23. Ishikawa H, Takaki A, Tsuzaki R, Yasunaka T (2014) L-Carnitine Prevents Progression of Non-Alcoholic Steatohepatitis in a Mouse Model with Upregulation of Mitochondrial Pathway. PLoS One 9(7): e100627.

24. Demiroren K, Dogan Y, Kocamaz H, Ozercan IH (2014) Protective effects of L-carnitine, $\mathrm{N}$-acetylcysteine and genistein in an experimental model of liver fibrosis. Clin Res Hepatol Gastroenterol 38(1): 63-72.

\begin{tabular}{ll} 
BIOMEDICAL & Assets of Publishing with us \\
RESEARCHES & - Global archiving of articles \\
& - Immediate, unrestricted online access \\
\hline
\end{tabular}

